\title{
THERMAL TIMESCALE MASS TRANSFER IN BINARY POPULATION SYNTHESIS
}

\author{
Stephen Justham ${ }^{1}$ and Ulrich Kolb ${ }^{1}$
}

Studies of binary evolution have, until recently, neglected thermal timescale mass transfer (TTMT). Recent work has suggested that this previously poorly studied area is crucial in the understanding of systems across the compact binary spectrum. We use the state-of-the-art binary population synthesis code BiSEPS (Willems and Kolb, 2002, MNRAS 337 1004-1016). However, the present treatment of TTMT is incomplete due to the nonlinear behaviour of stars in their departure from gravothermal 'equilibrium'. Here we show work that should update the ultrafast stellar evolution algorithms within BiSEPS to make it the first pseudo-analytic code that can follow TTMT properly. We have generated fits to a set of over 300 Case B TTMT sequences with a range of intermediate-mass donors. These fits produce very good first approximations to both HR diagrams and mass-transfer rates (see figures 1 and 2), which we later hope to improve and extend. They are already a significant improvement over the previous fits.

Our work shows that naive use of the whole star Kelvin-Helmholtz timescale should be avoided in modelling TTMT: by definition it is invalid in TTMT as the star can no longer be considered as responding holistically - see also Langer et al.,2000 (A\&A 362 1046). The success of our fits indicates that pseudo-analytic fit-based population synthesis can be extended to treat Case B TTMT; we can reproduce mass transfer rates and luminosity changes with simple fitting formulae. Moreover, our fits vary systematically in parameter space; understanding the reasons for these variations should lead to a better understanding of TTMT. Including the extent to which mass transfer is conservative in our parameter space (see figure 3) means our final set of fits will be very flexible. We intend to complete the coverage of Case B mass transfer and move onto Case A systems; the fits will be added to BiSEPS and used to investigate LMXB and CV evolution.

\footnotetext{
${ }^{1}$ Department of Physics and Astronomy, The Open University.
}

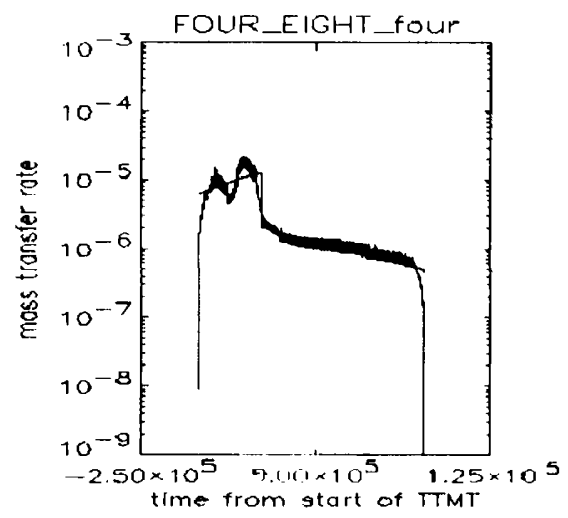

Fig. 1. A typical mass transfer rate fit.
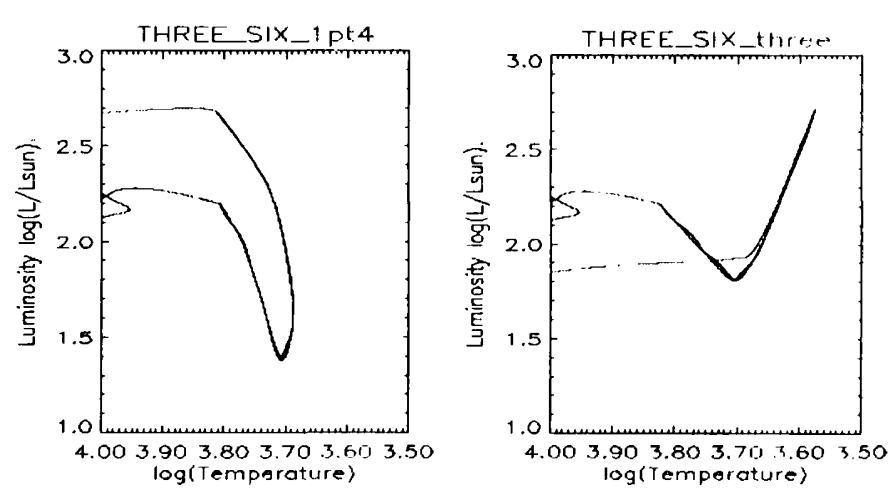

Fig. 2. Two examples of HR fits; both systems initially have a $3 M_{\odot}$ donor and a six day period.

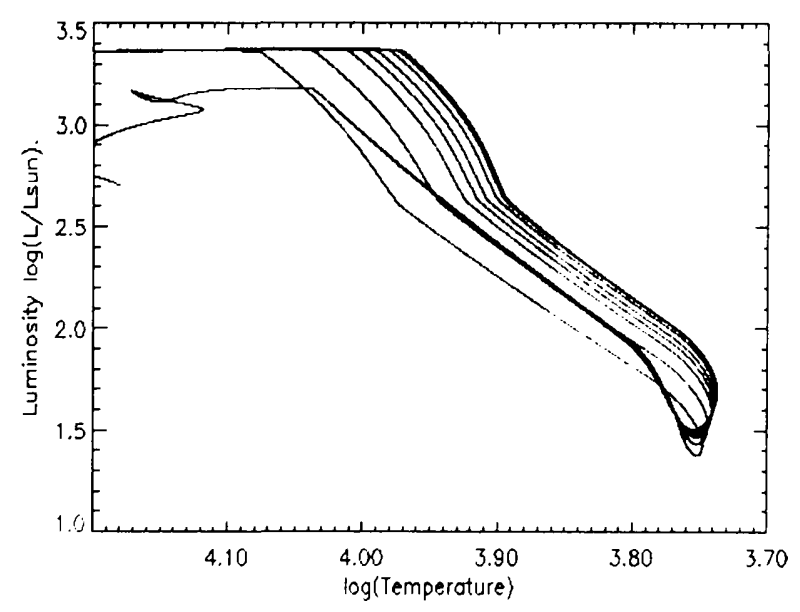

Fig. 3. The spread of $\mathrm{HR}$ evolutions as our mass transfer prescription alters between conservative and nonconservative for a system initially containing a $5 \%$ donor with a 5 day orbital period and a $2 M \circ$ accretor. 